\title{
Dynamic routing and resource allocation in WDM transport networks
}

\author{
Jan Späth * \\ Institute of Communication Networks and Computer Engineering (IND), University of Stuttgart, Pfaffenwaldring 47, D-70569 Stuttgart, \\ Germany
}

\begin{abstract}
This contribution concentrates on dynamic routing in Wavelength Division Multiplexing (WDM) networks. It is shown that a strategy based on pre-calculated alternatives and an adaptive dynamic path search performs very well over a wide load range. Moreover, specific effects for the impact of resource allocation strategies in photonic WDM networks are highlighted, especially the influence of wavelength converter usage strategies in networks with partial conversion. The paper also investigates the way non-Poisson traffic behaviour affects performance of routing strategies and presents how the results from dynamic routing investigation can help to optimise the network planning process. (C) 2000 Elsevier Science B.V. All rights reserved.
\end{abstract}

Keywords: Optical transport network; WDM; Routing strategy; Dynamic routing; Performance evaluation

\section{Introduction}

Wavelength Division Multiplexing (WDM) technology is globally used to cope with rapidly increasing bandwidth demands in telecommunication networks, especially for wide-area transport networks. At the moment point-to-point systems are already widely installed by many network operators and (all-) optical networks including optical routing and switching are approaching. Many different architectures and application areas are described in the literature most of which are based on the introduction of a WDM transport layer $[35,37]$. Two basic schemes are distinguished in the

\footnotetext{
* Tel.: +49-711-6857990; fax: +49-711-6857983. WWW: http:/l www.ind.uni-stuttgart.de/ $\sim$ spaeth.

E-mail address: spaeth@ind.uni-stuttgart.de (J. Späth).
}

literature. Either there is no wavelength conversion in the network - also called Wavelength Routing (WR) or Wavelength Path (WP) concept - or there is full wavelength conversion in the network - also called Wavelength Interchanging (WI) or Virtual Wavelength Path (VWP) concept [36]. Recently, several concepts with partial or limited wavelength conversion have also been proposed due to the high cost and still uncertain benefit of wavelength converters. Main concepts comprise either a limited number of converters in a node [26], or a limited number of nodes equipped with converters [43], or limited range conversion where converters cannot convert from any input wavelength to an arbitrary output wavelength [53].

The ever increasing transmission capacity of optical fibres leads to large transport streams in the core network. At the moment, WDM networks are used for transporting a large number of low bit rate streams. Moreover, dynamic operation of a 
WDM network layer still suffers from technological and management problems. Therefore, today's WDM networks usually provide "permanent" channels which are only manually reconfigurable or even completely static. For that reason, many studies and investigations were performed aiming at the optimal design and dimensioning of a WDM layer for static traffic requirements. These investigations comprise various Static Routing and Wavelength Assignment (static $R W A$, also called Static Lightpath Establishment, SLE) problems. Usually, a main goal of such a task is to minimise the required number of wavelengths or fibres in the network, or to show possible improvement by using wavelength converters (see for example many contributions in $[9,17]$, including a good overview in [21]).

However, in the near future increased dynamism can be expected for the WDM layer due to several reasons. The bit rates available for end users will further increase due to new broadband access techniques (such as $x$ DSL) which are being introduced now by various network operators [13]. In the future, there will be more users connected to telecommunication networks and an increasing portion of these users will not only use voice services, but also data services with higher bit rates. This leads not only to higher requirements for access networks, but also to more dynamics in the core network.

Moreover, with the increasing use of high bandwidth services in the Internet higher dynamics of traffic pattern changes will be observed. In addition, there is a lot of work going on to bring the Internet Protocol (IP) layer directly on top of a WDM layer in so-called "IP over WDM" networks $[1,32,51,52]$. This stresses the importance for transport networks to be able to rapidly adapt to new traffic patterns. A possible future scenario could therefore be a dynamic WDM layer directly controlled by IP routers which are able to request and release wavelength paths to distant routers. This would result in high dynamics for long-haul WDM paths. Similar influence on the WDM layer results from "Optical Burst Switching" ideas as described, e.g., in [32]. Such a network concept could also result in increased dynamism for wavelength paths.
Furthermore, new services can be provided by a WDM layer. This comprises high bit rate protocoltransparent corporate networks and "carrier's carrier" services, maybe even with the provisioning of transparent wavelength paths. Moreover, due to the huge bit rates in such networks, advanced protection and restoration mechanisms which also require some dynamics in the network become very important. Finally, in addition to this expected increase of dynamics it has to be considered that the impact of single blocking events in the WDM layer (e.g., blocking of a wavelength path request) on revenue loss for operators and on service outage for end users is drastically increasing due to the huge bit rates carried on each wavelength.

Due to these reasons the investigation of $\mathrm{dy}$ namic network behaviour for WDM networks becomes more important. These investigations should be combined with static planning and dimensioning tasks as described above (see Fig. 1). Only such a comprehensive approach allows achieving a network solution which is optimal not only for the static requirements considered during the planning phase, but also the dynamic situation the network will face during operation.

In the current literature, much work can be found dealing with dynamic routing in WDM networks (often also called Dynamic $R W A$, or Dynamic Lightpath Establishment, DLE). First of all, many analogies exist to dynamic routing in "classical" electronic switching networks [3,14]. However, several specific aspects have to be considered for WDM networks additionally. A recent overview describes especially the influence of

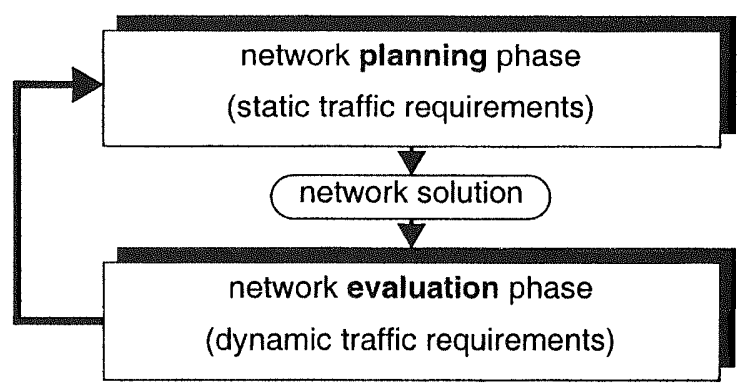

Fig. 1. Two main phases of a comprehensive network planning process. 
wavelength conversion but investigates also the influence of topology, routing strategy, and several technological parameters, and contains various references to related work [54]. Other overview papers are Ref. [21] which presents many references dealing with wavelength conversion gain for static and dynamic traffic conditions, and Ref. [33] which describes wavelength conversion technologies and several analytical methods to evaluate performance benefits of wavelength converters. In [34] an approach is shown to develop upper bounds for many static and dynamic RWA algorithms. The influence of wavelength converters on network throughput for both, SLE and DLE is the main focus of Ref. [49]. Other work considers the influence of different protection schemes on dynamic routing performance [42], focuses on effects in ring networks [29], or describes analytical models for certain approximations [6,7].

Most papers dealing with dynamic investigations for WDM networks are based on Poisson traffic assumptions due to the uncertainty concerning real traffic behaviour in future WDM networks. Although various traffic models are known in literature [2], it is still unclear whether they can be used for the description of traffic in a WDM transport layer. Several measurements in other networks have documented that some connection arrival processes are still well-modelled as Poisson processes [31], whereas some packet arrival processes in wide area networks [31] or Internet dial up behaviour [11] show non-Poisson behaviour. Thus recently, different non-Poisson traffic models were also used for investigating WDM networks. These studies show that in many cases routing performance is influenced by traffic behaviour $[29,39,40,45,54]$. Therefore, this work also considers different traffic characteristics.

This paper extends work presented in $[40,41]$. It concentrates on the dynamic routing and wavelength assignment problem and comprises Poisson as well as non-Poisson traffic assumptions. It considers randomly arriving wavelength path requests which are released again after a random holding time (called "path holding time" in the following). An application area is therefore a future core network with a meshed structure carrying large traffic streams with high dynamics. All performance investigations reported in this paper are done by simulation studies owing to the fact that all known analytical approaches require many simplifications and approximations, e.g., for routing strategies or traffic assumptions $[6,7,16,24$, $43,47]$.

Section 2 describes the large problem complexity due to many input parameters and their complex interrelations. These parameters define the degrees of freedom for routing strategies. In Section 3 a modelling approach is introduced and a simulation tool based on this modelling is shortly described. Section 4 presents a number of results derived with this tool. This comprises a comparison of several routing strategies with a different number of alternatives, dynamic on-line path search (i.e., at the moment of a path request), and adaptive length limits to restrict path lengths. Following that, the strong influence of converter usage strategies for networks with partial wavelength conversion is shown. In Section 4.4, nonPoisson traffic characteristics are used as a firststep towards better understanding the influence of traffic behaviour on the developed routing strategies. Finally, Section 4.5 presents an example for an efficient and focused network capacity upgrade based on results from dynamic investigations. Such an iterative approach may help to optimise a comprehensive network planning process that also considers dynamic network operation.

Throughout the paper, a wavelength channel corresponds to a wavelength on a single link whereas a wavelength path (also called lightpath in literature) describes a concatenation of wavelength channels, possibly with some wavelength conversion in between. Finally, a route describes the geographical way of either a wavelength channel or path.

\section{Parameters for the dynamic routing problem}

There are many different parameters which influence network performance under dynamic traffic conditions. Many of these parameters are interdependent: the impact of one parameter on blocking may depend upon the value of another parameter. This leads to the conclusion that one 
has to be careful with results obtained by variations of only one parameter. This section gives various examples for parameters and describes the assumptions made for the studies in the following sections.

\subsection{Network topology and dimensioning}

A network topology can be modelled as a graph, which in general is directed and weighted. This graph plays a very important role for routing investigations since it determines many parameters which directly influence a routing strategy. One example is the diameter of a network which is usually defined as the longest "Shortest Path" for all node pairs, i.e., the largest distance occurring in the graph. Arbitrary measures can be used for describing a path length, e.g., geographical distance, cost, or number of hops.

Another important parameter defined by the network graph is the number of disjoint (i.e., physically independent) alternative paths available for a node pair. In more detail, node disjoint and link disjoint paths can be considered. For both cases, one can further distinguish between fully or only partially disjoint paths. Moreover, also the relation of original and alternative path lengths is an important indication for additional resources required by alternatives. A routing strategy should consider the distribution of path lengths for original as well as for alternative paths, i.e., the number of one hop paths, two hop paths, etc.

The dimensioning of the network is another important parameter closely related to topology. It comprises dimensioning of links (e.g., number of fibres) as well as nodes (e.g., number of available switching elements) and has a strong influence on network performance parameters. The dimensioning strongly depends on traffic parameters (see Section 2.5).

Several investigations reported in literature focused on the influence of network topology (e.g., see $[5,18,28]$ for different variants of the static RWA problem and [30,49] for studies on certain aspects of dynamic routing). The results shown in the following will not concentrate on this but will demonstrate that there are other parameters with a strong influence on network performance. How- ever, to confirm that the achieved results and conclusions are valid for a broad spectrum of topologies various different example networks were investigated. But to allow an easy comparison of results only two example networks shown in Fig. 2 are used for all studies presented in this paper.

For these networks, which could both represent German national transport networks, an appropriate dimensioning is needed in order to be able to do performance evaluation under dynamic conditions. However, the focus of this paper is not on the static dimensioning phase (recent work related to this is contained, e.g., in $[9,17])$ but on the evaluation phase considering dynamic traffic conditions. Therefore, the networks were dimensioned using a simple planning process that is mainly based on Shortest-Path routing. In addition, an optimization step was performed to free links and fibres with low utilization by re-routing appropriate paths on alternative routes.

Dimensioning was done for symmetrical traffic demands derived from a simple model taking population and distance of a node pair into account. This demand is called static traffic demand and network load for dynamic investigations is expressed in relation to this demand. An 8 channel WDM system was assumed for all fibres in the network (leading to multiple fibre links with different number of fibres per link) and therefore all case studies shown in this work were performed for 8 channel WDM systems on all fibres.

The network solutions differ with respect to various parameters. A comparison of some key characteristics resulting from the applied dimensioning process is given in Table 1. For the de-

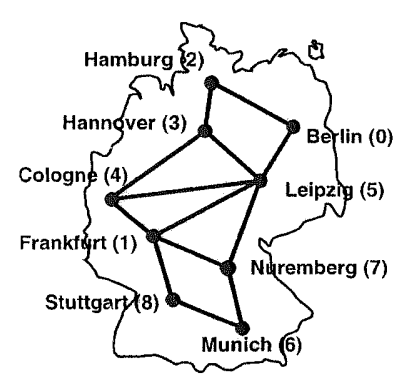

a) 9-node network

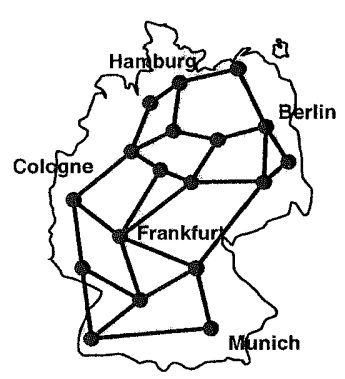

b) 18-node network
Fig. 2. Example networks used for studies in this work. 
Table 1

Characteristics for example networks as a result of the dimensioning process

\begin{tabular}{|c|c|c|}
\hline & 9-Node network & 18-Node network \\
\hline Number of nodes & 9 & 18 \\
\hline Number of links (ducts) & 13 & 29 \\
\hline Meshing degree & 2.89 & 3.22 \\
\hline Network diameter & 4 & 6 \\
\hline Total number of fibres & 218 & 930 \\
\hline Number of wavelength paths & 944 & 1612 \\
\hline
\end{tabular}

" $\Sigma_{\text {atl nodes attached links }) / \text { number of nodes. }}$

"Number of links (hops) for longest "Shortest Path" taking node distances into account.

"Total number of end-to-end wavelength paths for dimensioning = mean number of path requests for $100 \%$ network load for dynamic investigation.

velopment of several routing strategies topological network parameters were considered in detail (see Section 4.2).

\subsection{Node functionality}

The node functionality - and related to this the network architecture - determines the possibilities available for a routing process. The two basic node types of specific interest for WDM networks are Cross-Connects (CCs) and Add/Drop Multiplexers (ADMs). For both node types there are many variants with different functionality concerning switching (e.g., internal blocking), wavelength conversion (no conversion, full conversion, or partial conversion), or regeneration (which is often related to conversion since e.g., opto-electronical regenerators may incorporate conversion capabilities).

There are also many realisation approaches leading to the same functionality. For example, the CCs developed within the European ACTS projects OPEN and PHOTON [10] or the CC described in [23] can be equipped in such a way that they achieve identical functionality with different architectures. Refined node models are required when protection and restoration methods or additional technological or physical limitations are taken into account.

However, implementation details are of less importance for network performance investigation which only takes node functionality into account. This allows a simplified modelling based on CC nodes for the studies in this paper. Section 3 describes the modelling approach in more detail.

\subsection{Transmission functionality}

There are also many technological parameters describing the transmission functionality which influence routing performance. An important example is the available bandwidth for a link. For WDM networks, the total capacity results from the bit rate per wavelength channel multiplied by the available number of wavelength channels per link. The latter is defined by the number of fibres on a link multiplied by the number of wavelengths available on a fibre. In many scenarios (especially when there is not full conversion in the network), not only the total capacity is important, but also in which way this capacity is achieved, i.e., the actual values for bit rate, number of wavelength channels, and number of fibres. This study considers only the "wavelength level", i.e., each path request requires a full wavelength path.

Another technological parameter is the transmission distance achievable for a certain bit rate. Related to this are regenerator requirements to achieve a certain transmission performance. These parameters have a strong influence on network cost, but they may also influence routing functionality (for example, some long routes may not be realisable although resources are available).

\subsection{Strategies for the routing process}

Obviously, the routing strategy plays a central role for network performance under dynamic traffic conditions. Many different "basic" routing strategies can be distinguished, such as shortest-path routing, or least-loaded link routing. In 
addition, there are various search strategies for allocating network resources which also can influence network performance.

For routing in WDM networks, many wellknown strategies from electronic switching networks can be adapted, but several specific features of WDM networks have to be taken into account. Some examples are given in the following, whereas in Section 4 several strategies are developed and investigated in detail.

WDM networks with multiple fibres on a link provide an additional degree of freedom for channel assignment due to the availability of space and wavelength (or frequency) dimension. It is possible either to search along all fibres for one wavelength before changing to the next wavelength, or to search along all wavelengths on one fibre before going to the next fibre (see Fig. 3). For the search order there are also different possibilities such as first fit, sequential, or random search. In WDM networks with partial or no conversion these strategies usually lead to different network performance whereas in the case of full conversion there is no difference.

The mentioned wavelength dimension also leads to an additional degree of freedom for routing decisions. To route a path between a node pair, in addition to the way also a wavelength has to be chosen. This allows for example two strategies called "path priority" and "wavelength priority" (see Fig. 4). The "wavelength priority" strategy performs a search among all possible routes for a given wavelength before trying another wavelength (i.e., colour), whereas the "path

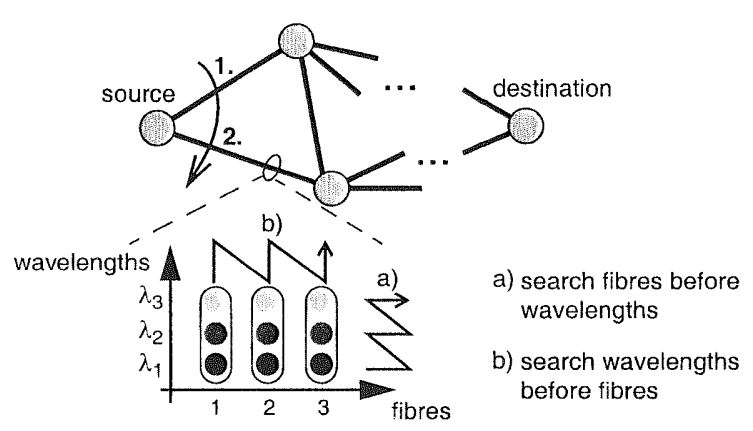

Fig. 3. Search strategies for route and channel allocation in a WDM network.

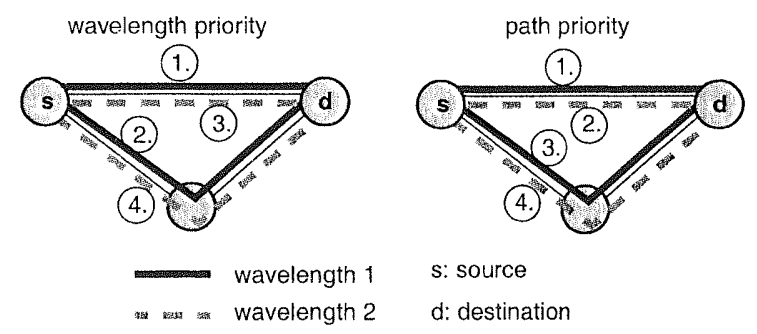

Fig. 4. Priorities for routing decisions.

priority" strategy checks all wavelengths on a given route before trying another route. These strategies can result in different network performance for networks with restricted or no conversion capability and were investigated in [38].

Another example are converter usage strategies which are important for networks with partial conversion. In this case, there is often a trade-off between the length of a path and the required number of converters. For the example shown in Fig. 5 there are two options for establishing a path between nodes $s$ and $d$ : there is a short route (2 hops) which requires a converter due to already occupied wavelength channels along this route, and there is a longer route ( 3 hops) which does not require any conversion. It was already demonstrated that different converter usage strategies have a strong impact on the achievable network performance and that there are situations in which more converters lead to a blocking increase due to disadvantageous routing decisions [39]. Section 4.3 will investigate this aspect in more detail.

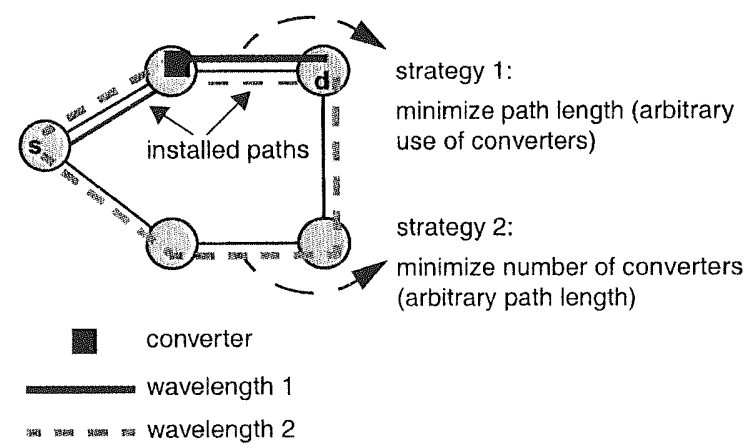

Fig. 5. Different strategies for the trade-off between converter usage and path lengths. 


\subsection{Traffic parameters}

The traffic assumptions made for a routing investigation have a strong influence on performance results. It is always difficult to find traffic descriptions which are on one hand accurate enough to cover all effects, but are on the other hand as simple as possible to simplify the analytical calculations or the required software tools for an investigation. The following parameters can be distinguished for a traffic description:

- Traffic volume: This parameter describes the absolute traffic values. For WDM networks, it usually is expressed in terms of the number of wavelength paths needed between each node pair.

- Traffic distribution: This parameter describes the distribution of traffic among the network nodes. Common examples are a uniform distribution (e.g., the same traffic between all node pairs) or a distance dependent traffic pattern (e.g., decreasing traffic with increasing node distance).

- Traffic characteristics: This parameter describes the behaviour of traffic streams. For this description many different models known from literature are available [2]. To characterise traffic behaviour for circuit switched (telephone) networks, very often the two parameters call interarrival time and call holding time are used [14]. In the following, this approach is also applied by describing these two parameters with their mean value and their coefficient of variation (see Section 3).

For WDM networks, the traffic description problem is further complicated because there are not enough measurements available up to now. Therefore, many work on dynamic behaviour of WDM networks reported in literature is based on a simple Poisson traffic model (see for example $[15,49,55])$. Recently, also various non-Poisson models for WDM networks were introduced but these models are not based on real measurement data (see for example $[29,39,45,54]$ ). However, it is well-known from many traffic theoretical work that traffic behaviour strongly influences network performance. Therefore, results have to be checked if better traffic models become available. In Section 4.4 , this point is investigated in more detail and the influence of path interarrival and path holding time parameters on the routing performance is demonstrated.

\section{Modelling approach and tool realisation}

This study concentrates on the WDM network layer by considering routing of individual wavelength paths, i.e., each path request requires one end-to-end wavelength path (which may include wavelength conversion). Concerning ITU-T Recommendation G.872 (former G.otn) for optical network architecture, this corresponds to the $O p$ tical Channel Layer Network [19]. Thus, neither physical transmission aspects nor higher network layers such as SDH or other client layers are taken into account. Moreover, the network is assumed to be in a "normal operation" state, i.e., no failure events and corresponding restoration mechanisms are considered.

The network model is shown in Fig. 6. Network nodes are connected via links which contain an arbitrary number of fibres (according to the network planning phase that has to be performed before a simulation). At each node, traffic may originate or end. One traffic generator for each possible node pair is used to model traffic between a source-destination node pair. This generator describes the traffic behaviour using two parameters:

- interarrival time between two wavelength path requests of a node pair;

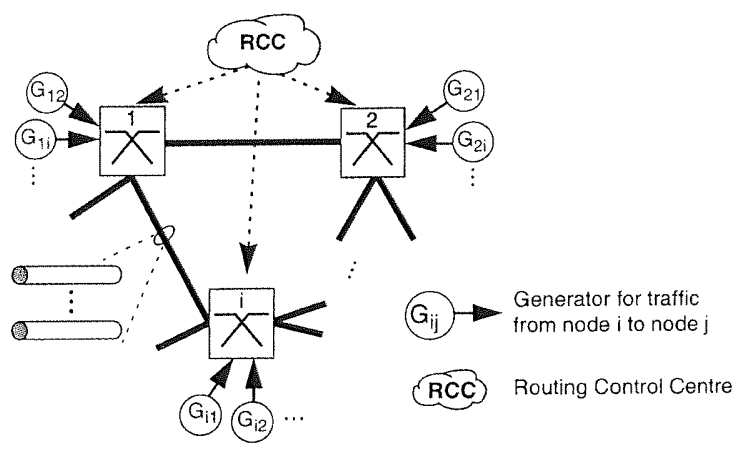

Fig. 6. Network model with traffic generators and crossconnect nodes. 
- path holding or service time, i.e., the time a wavelength path remains established.

For the studies in this paper, first exponential time distributions for interarrival times (corresponding to a Poisson traffic behaviour) and path holding times are used. To investigate the influence of traffic behaviour, other traffic characteristics are investigated in Section 4.4.

All studies in the following consider symmetrical, bidirectional traffic (which is still the usual case in today's transport networks) where paths for both directions are routed along the same way. Other path types such as unidirectional paths or bidirectional paths with separate routing for both directions could be realised by small extensions of the model. Path handling is as follows: a path request is randomly generated by an end node. If the path cannot be established, it is lost (no special repeat behaviour is considered). If the path can be established, the corresponding wavelength channels are released after a random time span following the path holding time distribution.

It is assumed that routing is managed by a central routing instance (Routing Control Centre, RCC). The RCC has complete knowledge on link and node states. This model corresponds in reality to central routing (with a real $\mathrm{RCC}$ ) or to source routing (with each node having a full picture of the whole network). This approach seems feasible since in the foreseeable future WDM networks will be widely managed by a central instance. In earlier work, a model and several strategies were also developed for decentralised routing where nodes have only a local view of the network $[38,39]$.

The node model is shown in Fig. 7. Basically, the nodes are assumed to have cross-connect functionality. Multiple input and output links each equipped with an arbitrary number of fibres are attached to a node. Additionally, wavelength paths may start or end at a node. The space switching matrix is assumed to be non-blocking. However, the conversion capability of a node may be limited. All nodes have a "share-per-node" structure where all converters are located in a converter pool which is shared by all wavelength paths passing through the node [26]. An empty pool corresponds to the WR (WP) case, a pool with as many converters as incoming wavelengths

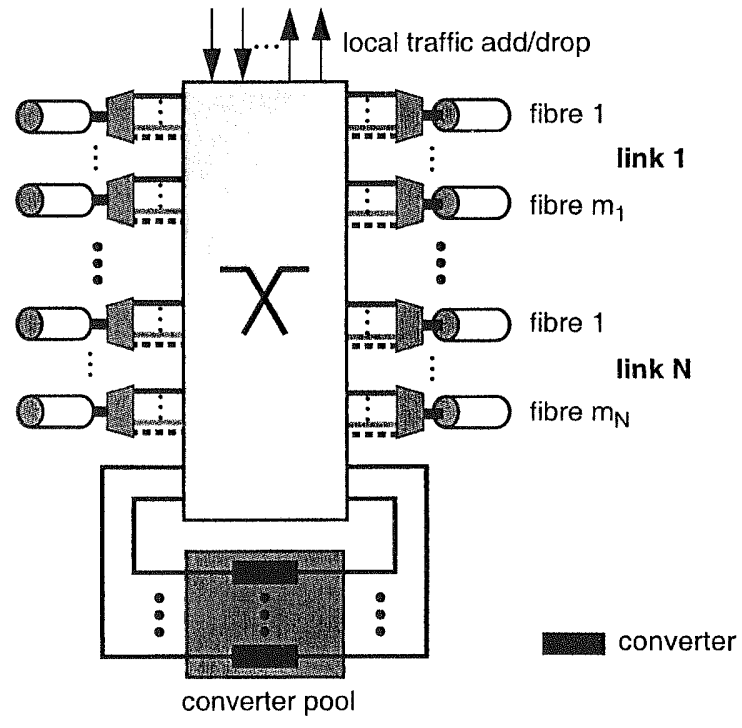

Fig. 7. Model for a network node with a "Share-per-Node" converter pool.

corresponds to the WI (VWP) case. By using a number of converters in between, partial conversion is realised. Furthermore, it is assumed that all converters are able to switch from any incoming wavelength to any outgoing wavelength. In the following, the conversion degree (in \%) is introduced to describe the converter pool equipment. For example, with a conversion degree of $25 \%$ the number of converters in the pool corresponds to $25 \%$ of the number required for the WI (VWP) case. It has to be noted that due to this definition a node with many input fibres has a higher number of converters than a node with few fibres for the same conversion degree.

To perform quantitative studies, a discrete event simulation tool based on the model described above has been implemented. The tool uses a complex object-oriented simulation library that supports simulations in many ways, e.g., by providing modules for simulation control, traffic generators, and statistical evaluations [22]. An important design criterion for the tool is a flexible and modular structure which has been achieved by an appropriate object-oriented design. In this way further enhancement of the tool, e.g., by defining new routing strategies is simplified. Moreover, the 
tool allows a flexible handling of input parameters such as network topology, traffic description, or routing strategy parameters.

The tool was realised in $\mathrm{C}++$ and can be executed on several platforms. It delivers a large variety of results which are usually given as mean values with confidence intervals. Examples are total network blocking, blocking of an end-to-end node pair, converter occupancy in a node, mean path lengths for successful path establishments, and lengths of alternative routes compared to the original "first way". The following section presents several case studies performed with this tool.

\section{Case studies and results}

First, an adaptive dynamic routing strategy with "biased" alternatives is developed and the performance of this strategy is compared with some simpler strategies. Then, the influence of additional length limit strategies is shown before a specific effect for WDM networks - the influence of converter usage strategies - is presented. Section 4.4 investigates the influence of different nonPoisson traffic characteristics. Finally, it is demonstrated how simulation results can help to improve network planning in a very efficient way. All results are presented with $95 \%$ confidence intervals.

\subsection{An adaptive dynamic routing strategy with biased alternatives}

This section introduces the basic routing strategy used for the following investigations. Many routing strategies for traditional circuit-switched (telephone) networks are broadly investigated in literature (see for example in [3,14]). Many insights are still valid or can at least be transformed for WDM networks. In addition, several specific aspects have to be taken into account, mainly resulting from the influence of wavelength conversion.

First of all, routing performance strongly depends upon the number of alternatives allowed for routing a path request. Multiple alternatives reduce blocking significantly compared to the case where only a single route is allowed [15]. These socalled "alternative routing" schemes can be further improved by an additional route calculation at the moment of a path request $[3,20,30]$.

Furthermore, a routing strategy which considers the decisions and assumptions made during network planning, especially the static routing decisions, improves performance. Such schemes were also called "biased" routing schemes [48]. In the planning phase used for the case studies in this work, mainly Shortest-Path routing is applied to determine the necessary network dimensioning, and therefore dynamic routing is biased accordingly. However, such a scheme implies that the traffic patterns considered during planning and those considered during dynamic investigations are similar.

To show the influence of these elements the following basic routing strategies are compared [40]:

- Fixed Central Routing (FCR): This simple strategy allows only one fixed pre-calculated route for each possible path request. If no wavelength path (which may include wavelength conversion if possible) along this route can be found, the request is blocked.

- Alternative Central Routing on partially LinkDisjoint paths ( $A C R-L D)$ : This strategy provides for each shortest route (comprising $l$ links) between a node pair a set of alternatives which is developed as follows. One of the links of the original route is removed and in the resulting topology the shortest path between the node pair is calculated. This is repeated for all links of the original route resulting in $/$ alternatives. Finally, also a route completely disjoint to the original shortest route is added. Thus, there are in general $l+1$ alternatives, but depending on the network topology some of these alternatives may be identical. When a path request occurs, the path search with this strategy is done as follows. First, the shortest route is used. If this route is blocked, it is checked whether blocking occurred on only one link of the original route. In this case, the shortest route disjoint to this link is used. If this route is also blocked, or if multiple links on the original shortest route are blocked, the completely disjoint route is tried as last alternative. 
- Adaptive Dynamic Routing with x pre-calculated routes $(A D R x)$ : This strategy is the most complex strategy shown here and tries to incorporate all elements described above. It comprises $x$ pre-calculated routes for each node pair which are the $x$ shortest routes being completely disjoint among each other. In addition, it allows a dynamic (on-line) calculation of a new shortest path in case all pre-calculated routes are blocked. This dynamic path calculation can be adapted to the actual network state by using one or both of the following adaptation mechanisms. First, links loaded above a configurable threshold (which is described as number of occupied channels divided by the total number of channels on a link) can be excluded from the searchable network topology. Second, it is possible to consider length limits for alternative paths to avoid very long alternatives (see Section 4.2).

Fig. 8 shows some results for a specific case study which is representative for many studies which were performed with differing parameters. The horizontal axis represents the offered load (i.e., mean value of dynamic traffic) in relation to the so-called static traffic demand, which describes the static traffic demand assumed for network dimensioning. The parameter settings for Fig. 8 are as follows. The 18-node network is investigated for $0 \%$ and $25 \%$ conversion degree in the network

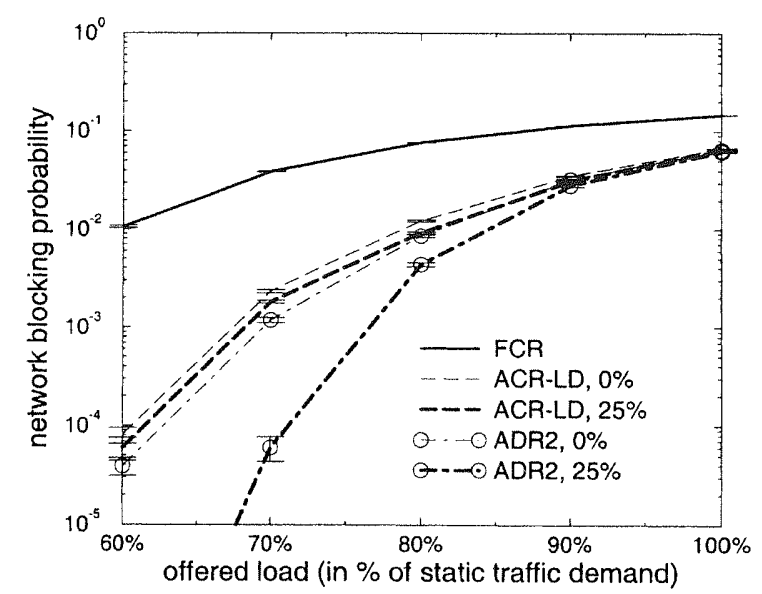

Fig. 8. Comparison of different basic routing strategies for the 18 -node network and two conversion degrees $(0 \% ; 25 \%)$. nodes. The ADR $x$ strategy is shown for 2 precalculated alternatives (ADR2) without any length limit and without considering a threshold for the dynamic path search. Other values for $x$ lead to similar blocking for the ADR $x$ strategy with a slightly higher blocking for ADR1, especially for a low conversion degree.

Main results from the figure are the following:

Influence of number of alternatives and dynamic route calculation: The results show that in principle all strategies which allow multiple alternatives perform much better than FCR for low network load. If the lines for a given conversion degree are compared (thin lines for $0 \%$ conversion, thick lines for $25 \%$ conversion), it can be seen that ADR2 achieves lowest blocking values over a broad load range. In the given scenario, ADR2 without conversion performs even slightly better than ACRLD with $25 \%$ conversion. This demonstrates the great importance and benefit of a dynamic path calculation with a central view on the network state.

Influence of offered load: It can be seen that the statements above are only valid for the "low load" area (up to a network load of approximately $85 \%$ of the static traffic demand in the case study), whereas for higher loads the different strategies converge. In other studies it was shown that for even higher loads an intersection of the curves for different strategies occurs and that FCR can achieve best performance [40]. This effect can be explained by looking to the path lengths (given in number of links) shown for the different strategies in Fig. 9. The mean number of links per path is reduced for the FCR strategy with increasing load because path requests for nodes with a large distance are more probably to be blocked. For the other strategies, mean length is increasing because some paths are accommodated along longer alternatives, but the price is that multiple short paths then might be blocked leading to higher overall blocking.

Influence of conversion degree: For the FCR scheme only one line is shown since the results for all conversion degrees (from $0 \%$ to $100 \%$ ) were very close together. The lines for $100 \%$ conversion degree are not shown in the figure because they are nearly identical to the lines achieved for $25 \%$ conversion for all strategies. Thus one conclusion 


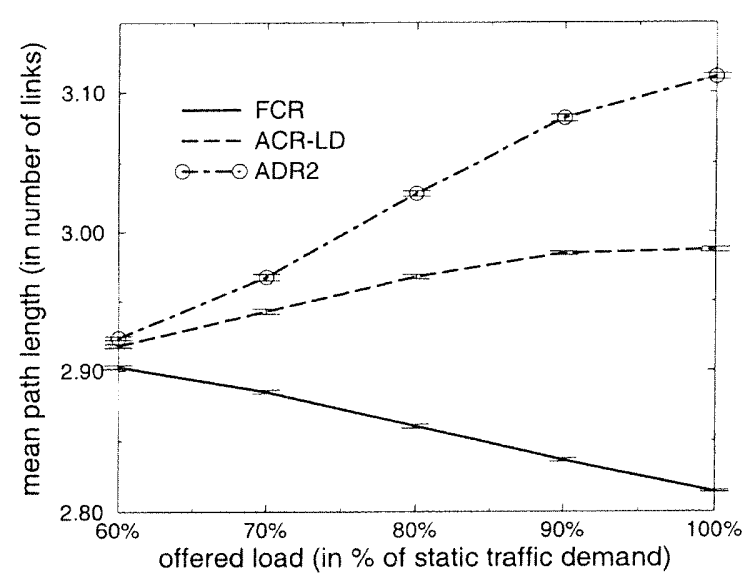

Fig. 9. Mean path length (in number of links) for the 18-node network and $25 \%$ conversion degree.

is that a small number of converters may improve network throughput under dynamic conditions, especially for strategies which allow multiple alternatives, but a $100 \%$ conversion provides almost no benefit compared to a smaller conversion degree. It has to be mentioned that the specific numbers for sufficient conversion or achievable throughput improvement strongly depend on almost all input parameters (e.g., topology, network dimensioning, routing strategy, traffic distribution). Section 4.3 will elaborate in more detail on the influence of partial wavelength conversion on network performance.

Finally, such studies allow to determine which mean load for dynamic traffic behaviour can be accepted in order to stay below a certain blocking probability. This mean load may have to be significantly below the static load used for network dimensioning. For example, in the case study shown here even with the best routing strategy the load must be lower than $70 \%$ of the static traffic demand in order to achieve a network blocking of less than $10^{-4}$. However, a routing strategy also has to perform well for "overload" scenarios and therefore the following section describes an important strategy extension.

\subsection{Length limit strategies}

The above-mentioned behaviour for high load leads to the conclusion that some kind of addi- tional limitation strategy could be beneficial for the overall performance. This section introduces and compares several limitation strategies for the following representative scenario: the 9-node network with $25 \%$ conversion degree is investigated for the ADR2 strategy.

The most simple limitation strategy is to set an absolute limit for any path length. In Fig. 10 the influence of such a strategy is shown for several limit values. The number of hops is chosen as length parameter. It can be seen that a limit of 3 hops is too small since several node pairs are not able at all to establish a wavelength path. On the other hand, this limit leads to lowest blocking for very high load. Although the other limitation strategies are rather close together, it can be stated that a limit of 4 hops achieves good performance for very high loads, but results in higher blocking for low network load compared to a limit of 5 hops or the case without limit.

Thus, although an "absolute limit" strategy is simple to realise it has the following main drawbacks:

- there are unnecessary restrictions for low network load for which longer alternatives could be accepted without throughput degradation,

- unfairness occurs because node pairs with a short distance may still have many alternatives while in the worst case other node pairs may not be able at all to establish a path. This aspect

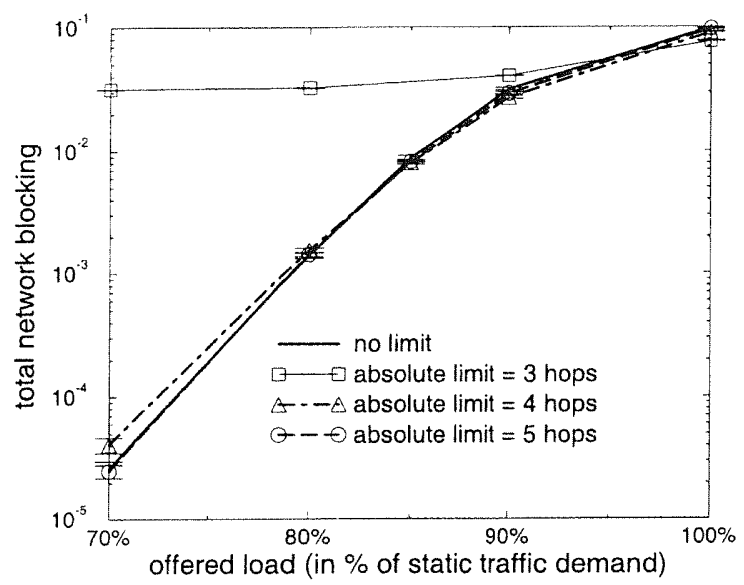

Fig. 10. Comparison of different absolute length limits for the ADR2 strategy (9-node network, 25\% conversion). 
is getting more critical with increasing network size due to increasing differences of node pair distances.

Therefore the following improved strategies are proposed:

- Relative length limit: With this strategy, length limits consider the length of the shortest route (i.e., the first alternative). Again, such a relative limit can be realised in various ways. After a detailed consideration of the network topology and traffic pattern an approach for the 9-node network has been chosen where the length of alternative routes is limited to $k_{i}$ times the original length of a route according to Table 2 .

- Adaptive length limit: This strategy combines the relative limits described above with a consideration of the load on network links. For an alternative route, a relative length limit is only applied if at least one link of the alternative route carries a load higher than a configurable threshold. Such an adaptive consideration could also be combined with various other strategies, e.g., an absolute limit strategy. Moreover, other threshold definitions are possible, e.g., by considering the average load of all alternative links. However, a difficulty occurs for WR networks. In this case the number of channels occupied on a link is no longer a sufficient measure due to the wavelength continuity constraint: a path request arriving at a link on a certain wavelength may be blocked although some other wavelengths are still free. Therefore, simple threshold mechanisms are especially useful with sufficient conversion.

Some results for the scenario described above can be found in Fig. 11. The relative length limit achieves a good performance for high loads but

Table 2

Parameters for the relative length limit strategy (9-node network)

\begin{tabular}{lll}
\hline $\begin{array}{l}\text { Number of hops for } \\
\text { "Shortest Path" }\end{array}$ & $k_{i}$ & $\begin{array}{l}\text { Maximum number of } \\
\text { hops for alternative } \\
\text { route }\end{array}$ \\
\hline 1 & 3 & 3 \\
2 & 1.5 & 3 \\
3 & 1.33 & 4 \\
4 & 1.25 & 5 \\
\hline
\end{tabular}

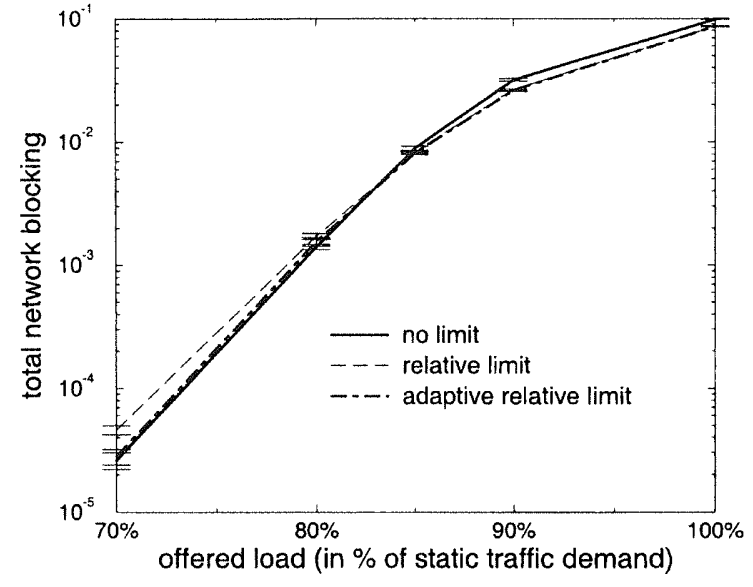

Fig. 11. Relative and adaptive length limits for the ADR2 strategy (9-node network, $25 \%$ conversion).

still shows unnecessary blocking increase for low loads. This is improved by using the adaptive strategy (with a threshold equalling $90 \%$ load on a link for the results in the figure). In conclusion, the results show that a biased, alternative, dynamic and adaptive strategy achieves very good performance results over the whole load range.

Moreover, another effect could be observed. For all studies without length limits blocking probability for traffic between a node pair increased with the node distance. The introduction of appropriately chosen length limits helps to improve the fairness in terms of a decreasing difference of blocking probabilities for paths between node pairs with different distances (similar to the definition in [4], where the difference between the node pairs with the shortest and the longest distance was used, respectively).

\subsection{Converter usage strategies}

WDM cross-connect networks offer several new degrees of freedom for routing decisions. In literature, the influence and interworking of different "wavelength selection" and "path selection" algorithms was already investigated for WR networks [30]. However, there are some more degrees of freedom which were only recently investigated [41]. In the following, the influence of the wavelength converter usage strategy is presented. This 
effect is specific for WDM networks and plays an important role in networks with partial wavelength conversion. Two different strategies are investigated:

- MinOff tries to minimise the path lengths while using converters arbitrarily (this strategy was used for all studies described above).

- MinOn tries to minimise the number of converters while accepting longer routes.

For the results in Figs. 12 and 13 the ADR strategy is investigated with 1 or 2 pre-calculated alternatives and no length limit for the 9-node network. Again, different load levels have to be

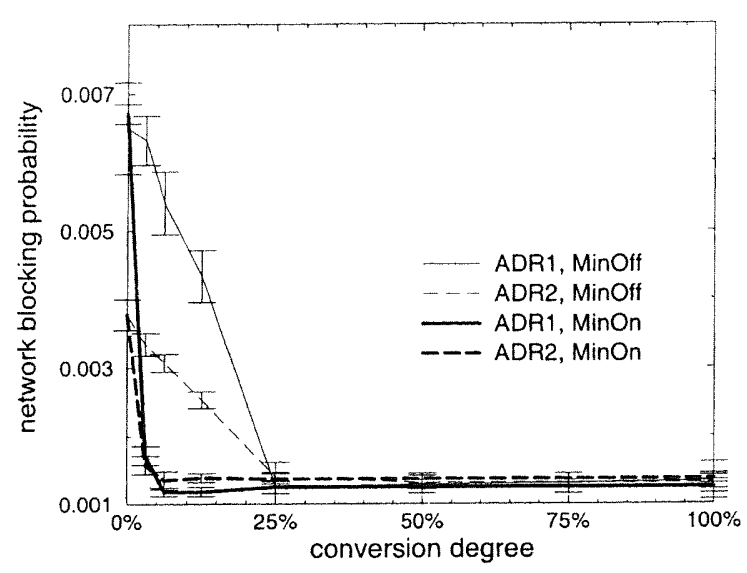

Fig. 12. Network blocking for different converter usage strategies and conversion degrees $(80 \%$ network load).

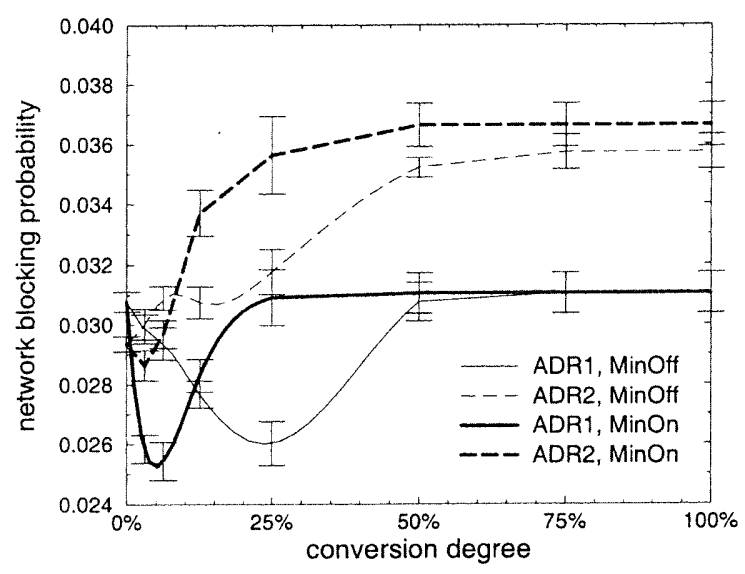

Fig. 13. Network blocking for different converter usage strategies and conversion degrees ( $90 \%$ network load). distinguished. Fig. 12 shows the network blocking probability versus conversion degree $(0 \%$ equals "no conversion", $100 \%$ equals "full conversion"). The figure is shown for a network load of $80 \%$ of the static traffic demand. In principle, already few converters decrease blocking significantly whereas for more than $25 \%$ conversion degree - which equals 2 converters per fibre - no further improvement can be seen (the occurrence of a local optimum for ADR1, MinOn for a conversion degree of approximately $6 \%$ is explained later).

So far, in literature similar results are already reported where performance is increasing with increasing number of converters, but the improvement rate is decreasing for higher conversion degrees (see for example $[8,46]$, or [43] where the strong influence of network connectivity is shown). There are also some results describing similar behaviour for various static network dimensioning problems. For example, in [27] a path accommodation algorithm is presented which tries to minimise the required number of converters for a given static traffic demand matrix. The optimization criteria is the number of cross-connect ports. One conclusion is that with an optimised algorithm about $10 \%$ conversion was sufficient to achieve the same cross-connect size as in the VWP case (although the specific number strongly depends on the actual case study parameters). In [44] an optimal converter placement is investigated for a WDM path and for WDM bus and ring networks. Again, for an optimised placement a rapid blocking reduction is achieved with some converters, whereas further increasing the number of converters leads only to small (in some cases even no) additional improvement.

But in addition to the effects described above, Fig. 12 also shows the strong influence of the Min On strategy for low conversion degree: starting from identical blocking values for "no conversion", blocking is quickly and significantly reduced with an increasing conversion degree and the optimum performance is already achieved with a conversion degree of only $\approx 6 \%$ (equalling 0.5 converters per fibre). For higher conversion degrees MinOn and MinOff strategies converge. Moreover, the results show that for low conversion degree ADR2 performs slightly better than 
ADR1, which is expected due to the additional pre-calculated biased route. For a higher conversion degree the results become comparable, in some cases ADR1 is even better than ADR2. This indicates that in these cases an "early" dynamic path search (i.e., already after the first alternative is blocked) is more efficient than using an additional pre-calculated route.

Whereas the behaviour shown in Fig. 12 is very similar for all load levels below $80 \%$, the situation changes for $90 \%$ network load (Fig. 13). This case reveals several effects very astonishing at the first glance. First, ADR2 leads now to higher blocking values than ADR 1 for a broad range of conversion degree. Second, only for a very low conversion degree additional converters reduce network blocking. For more converters, blocking is increasing, for ADR2 even above the value for 0 converters. The reason is that in a congested (heavy loaded) network more converters allow some longer paths which on the other hand may block several shorter paths. Although in general the difference between ADR 1 and ADR2 is larger than the difference between MinOn and MinOff, for low conversion degree the converter usage strategy still influences blocking significantly.

These results - which could be confirmed for many other scenarios - allow the conclusion that an optimal routing strategy for WDM networks has to be adapted to the available conversion capability of the network as well as the network load due to different behaviour for low and high network load.

Another interesting result is shown in Figs. 14 and 15 which present the converter occupancy given as mean value over time - in several nodes versus conversion degree for both strategies, $\mathrm{Min}$ On and MinOff. For these studies ADR2 was applied for the 9-node network shown in Fig. 2(a) with a load of $80 \%$.

Fig. 14 shows the converter occupancy for the MinOn strategy. The converter occupancy in border nodes is significantly lower than in central network nodes which is expected for the topology, traffic distribution, and routing strategy of the case study. Moreover, already for rather low conversion degree the converter usage reaches very low values. This changes significantly for the MinOff

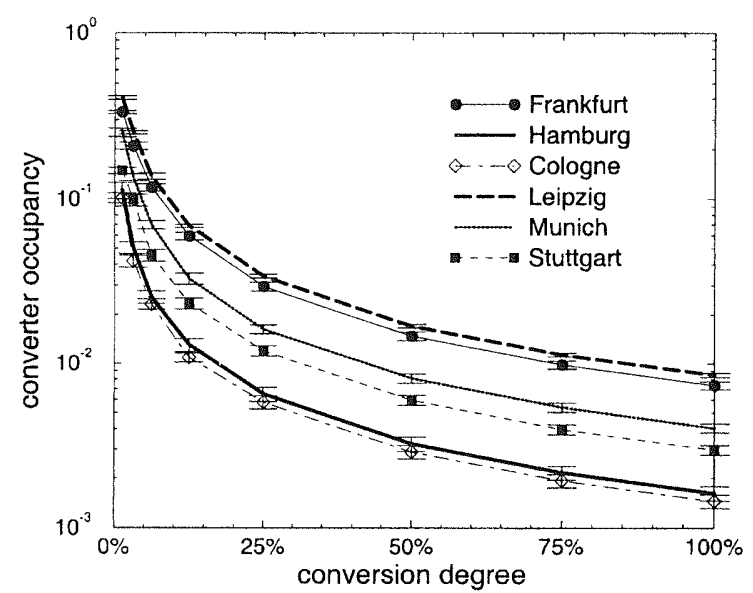

Fig. 14. Converter occupancy for ADR2 applied to the 9-node network with $80 \%$ load ( MinOn).

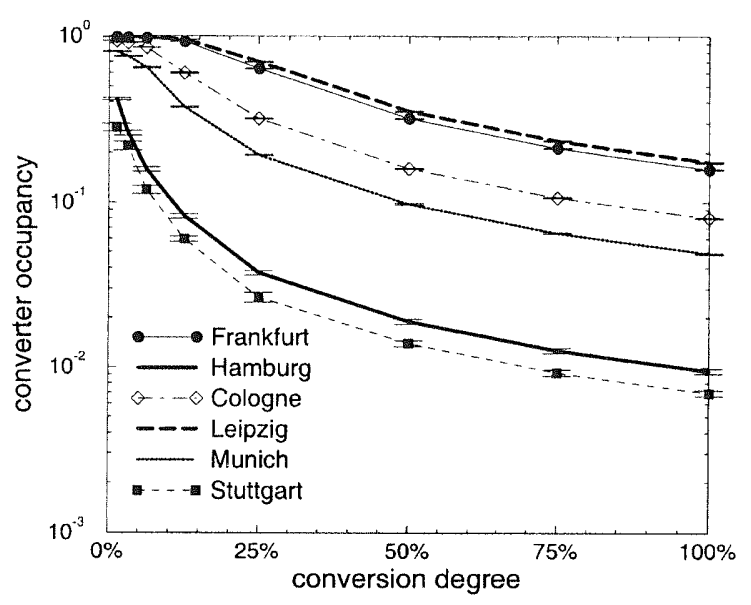

Fig. 15. Converter occupancy for ADR2 applied to the 9-node network with $80 \%$ load (Min Off).

strategy which leads to generally higher converter occupancy levels (Fig. 15). In addition, the relative order of nodes according to the occupancy level may change: e.g., Cologne has the lowest occupancy for MinOn but requires a rather high number of converters for MinOff due to the "greedy" converter usage strategy in this case. Thus, the actual converter requirement in a network node strongly depends not only on topology or traffic requirements, but also on the routing and converter usage strategy applied. 
Results like this - which where derived for many other scenarios including studies for decentral routing strategies [38] - allow an optimised network dimensioning by implementing wavelength converters at the optimum locations. This can help to further improve results from wavelength converter placement strategies, for which several heuristics were proposed recently $[44,46,50]$. But it is important to note that such a focused network upgrade not only depends on given input parameters as topology or traffic but also on the routing strategy and therefore has to be done specifically for each parameter configuration.

\subsection{Influence of Poisson and non-Poisson traffic characteristics}

Most investigations of WDM networks reported in literature are based on the assumption of Poisson traffic. Basically, there are two reasons for this:

- The approximation of real network traffic with Poisson arrival processes and exponentially distributed call holding times has been proven to be a good and analytically tractable approximation for many traditional (telephone) networks [14].

- Up to now there are no better or more accurate traffic models for WDM networks because no measurements are available for this kind of networks.

However, recent measurements for Internet dial up traffic have shown that traffic behaviour on the connection level can be significantly different from Poisson behaviour for various scenarios [11]. Traffic behaviour has a strong influence on network performance evaluation. Therefore, the question how routing strategies for WDM networks will behave under non-Poisson traffic is important and increasing work in this direction recently appeared although it is not clear at all how traffic in future WDM networks will behave. For example in $[29,45,54]$ different approaches to model non-Poisson traffic are reported by other authors. A detailed comparison of these models still has to be done.

In this study, non-Poisson traffic is generated as follows. The coefficient of variation $(\mathrm{CoV})$ for the interarrival and the path holding times is varied, respectively. With a negative-exponential distribution for the time variable a $\mathrm{CoV}=1$ is achieved. With hyper-exponential time distributions a $\mathrm{CoV}>1$ is realised similar to the approaches described in $[12,25]$. This type of distribution allows for example a simple description for bursty behaviour neglecting autocorrelation. A distribution with hypo-exponential behaviour is used to achieve a $\mathrm{CoV}<1$ [25]. This can be interpreted as a "more deterministic behaviour". In [39] the influence of non-Poisson traffic for hop-by-hop routing strategies was studied whereas in the following central strategies are investigated. The results shown below were derived for the 9-node network and the ADR2 strategy without length limits.

Fig. 16 shows the influence of the path holding time (i.e., service time) characteristics under the assumption of negative-exponentially distributed interarrival times, i.e., the path arrival process is Poisson. It can be seen that in this case the holding time $\mathrm{CoV}$ has almost no influence on network performance although the $\mathrm{CoV}$ is varied between 0 (corresponding to deterministic holding times) and 10 (which is a very high value for $\mathrm{CoV}$ ). The figure shows that this holds for a network without converters as well as for a network with full conversion capability. Moreover, the difference between the two conversion strategies is much more significant than between different $\mathrm{CoV}$ values.

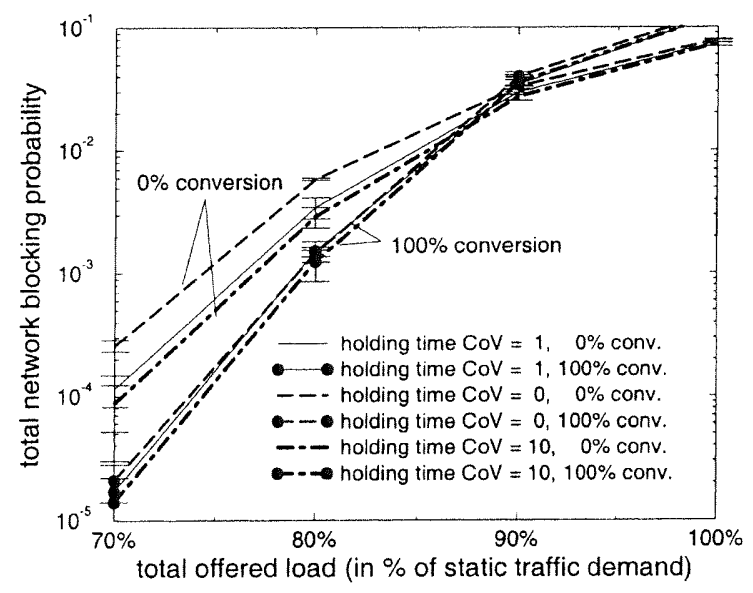

Fig. 16. Influence of holding time characteristics for a Poisson arrival process. 


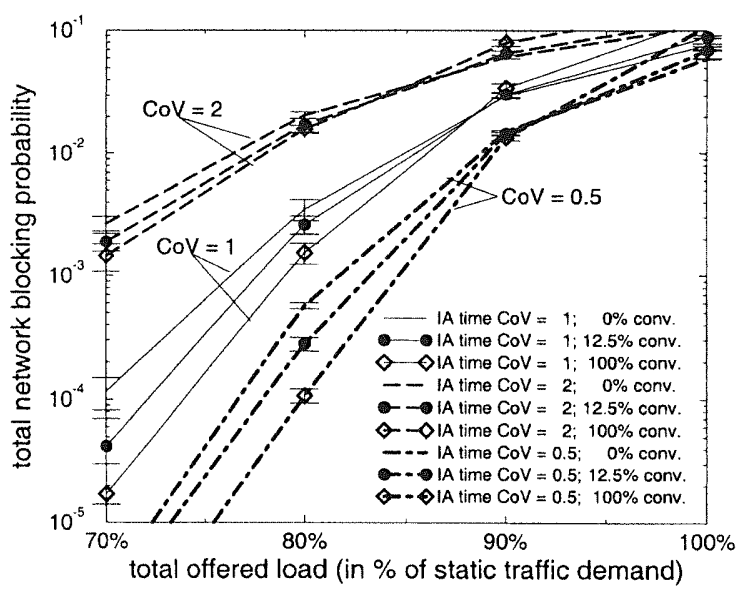

Fig. 17. Influence of interarrival time characteristics for negative-exponential path holding times.

The results are completely different if the interarrival time $\mathrm{CoV}$ is varied while path holding times are negative-exponentially distributed. Fig. 17 shows a strong influence of the interarrival time $\mathrm{CoV}$. Blocking probabilities are higher for $\mathrm{CoV}>1$ and lower for $\mathrm{CoV}<1$ compared to $\mathrm{CoV}=1$. This influence is much more significant than the difference between no, partial and full conversion capabilities.

Several cases were also investigated where both, interarrival and holding times are not exponentially distributed. In these cases, also the variation of the holding time $\mathrm{CoV}$ gets an important influence. Thus, only for a Poisson arrival process the path holding time behaviour did not show a significant influence on blocking. For all other cases a strong impact of traffic behaviour on performance results for WDM networks can be expected. Therefore, all results derived in literature for Poisson behaviour have to be revisited if traffic in future WDM networks will show different characteristics.

\subsection{Network dimensioning improvement using sim- ulation results}

Finally, this section shows how simulative network investigations for dynamic load can help to optimise link dimensioning. In the following, endto-end blocking probabilities for different node pairs are presented. The node numbers used in
Figs. 18 and 19 can be found in the network graph in Fig. 2(a). Each line describes the blocking of paths originating at the node assigned to this line and ending at the node depicted on the horizontal axes (lines are used to highlight which discrete points belong together). For the studies, ADR2 with minimisation of converter usage ( $\mathrm{MinOn}$ ) was applied to the 9-node network with a conversion

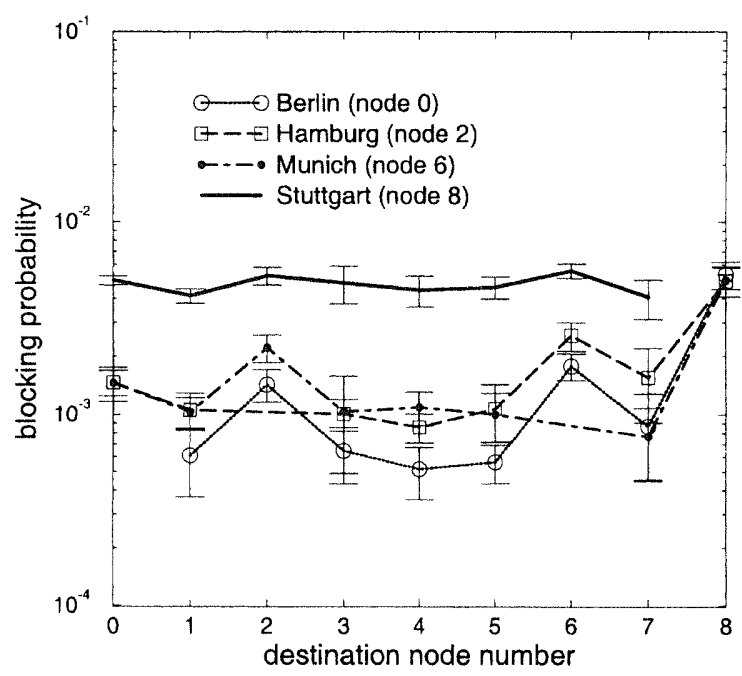

Fig. 18. End-to-end blocking without capacity increase (for ADR2 strategy with $\mathrm{MinO}$ applied to 9-node network loaded with $80 \%$ and $12.5 \%$ conversion degree).

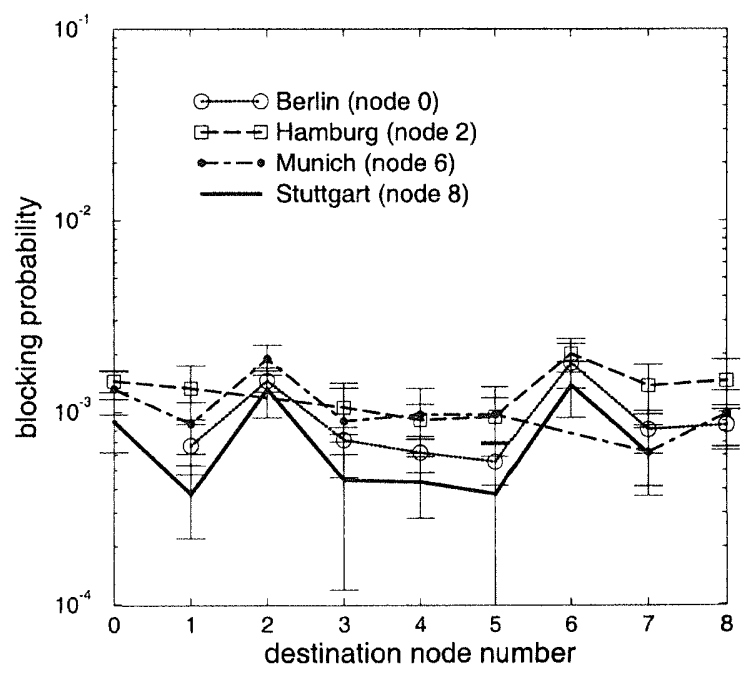

Fig. 19. End-to-end blocking with small capacity increase on one link (for ADR2 strategy with MinOn applied to 9-node network loaded with $80 \%$ and $12.5 \%$ conversion degree). 
degree of $12.5 \%$ ( 1 converter per fibre) and a network ioad of $80 \%$ of the static traffic demand. Poisson traffic with negative-exponential path holding times was used.

Fig. 18 gives the results for the "standard" network dimensioning (used for all studies presented so far) for selected node pairs. Only four lines are shown, all lines for the other nodes are in the same region or even below the lower three (broken) lines. The figure allows several conclusions which are specific for the case study performed and which were not obvious from the planning phase:

- For some destination nodes there is a tendency for higher blocking values compared to other nodes. In the example, nodes 2,6 , and especially node 8 show this behaviour.

- All paths starting in Stuttgart (node 8, solid line) experience a significantly higher blocking probability than the other paths.

This insight has been used for a selected network upgrade: the link between Stuttgart and Frankfurt has been equipped with one additional fibre for each direction. The result is shown in Fig. 19. Now, all node pairs are in the same region concerning blocking values. Moreover, the total network blocking was also decreased significantly: from $1.389 \times 10^{-3} \pm 6.9 \times 10^{-5}$ for the standard dimensioning to $7.44 \times 10^{-4} \pm 9.5 \times 10^{-5}$ for the case with improved dimensioning. This shows that already a very small (the two additional fibres correspond to a total network capacity increase of only $0.92 \%$ ) but well placed capacity increase can lead to a drastically network performance increase $(\approx 46 \%$ for the mean values). In addition, fairness with regard to differences in blocking probabilities - concerning different node pairs is increased as well: now, all node pairs experience similar mean blocking values.

Finally, it is remarkable that the right place for a capacity increase could not be found by looking to the blocking events occurring on a link. These statistics show no unusual behaviour of the link Stuttgart-Frankfurt. For various other links with similar number of blocking events a capacity increase did not lead to comparable throughput improvement (although some blocking reduction was achieved as well).

\section{Conclusions and outlook}

In this paper several routing strategies and specific effects for dynamic wavelength path routing in a WDM transport network based on crossconnects were investigated. Several cases for conversion capability in the nodes were considered, including partial conversion for share-per-node cross-connects. A new routing strategy was developed based on pre-calculation of "biased" alternatives (i.e., oriented according to the network design rules), a dynamic path search in case all alternatives are blocked, and an adaptive consideration of length limits to achieve good performance over the whole load range.

The influence of various parameters such as number of available alternative routes, offered load, and wavelength conversion degree on network performance was presented. Specifically, two strategies for wavelength converter usage were presented and compared. It was pointed out that converter usage strategies can be very important for networks with partial conversion. It was demonstrated that an increasing number of converters may lead to higher blocking if the routing strategy does not take into account conversion capability. This shows that for WDM networks there may occur unexpected effects which are partly new compared to traditional networks based on electrical switching, especially for low conversion degree and for high network load in WDM networks.

Moreover, the influence of traffic characteristics different to Poisson traffic was investigated. It turned out that in many cases the performance of routing strategies strongly differs for Poisson and non-Poisson traffic whereas for a Poisson arrival process the path holding time characteristic only has a small influence. From this follows that all results derived for Poisson traffic have to be checked if better models based on real network measurement become available in future. Finally, it was demonstrated that results from a dynamic network investigation provide a valuable feedback for a comprehensive network planning process that takes network operation into account. It allows avoiding unnecessary network equipment (shown for the number of wavelength converters in this paper), and achieving significant performance 
improvement already with small but well placed additional network capacities (shown for additional link capacity in this paper).

Further work could improve this insight by providing some kind of "automatic feedback loop" for the network planning process to take results from the investigation of dynamic behaviour for dimensioning of nodes and links into account automatically. Moreover, a better traffic description for various network scenarios (including transport of SDH networks as well as IP networks by the WDM layer) should be developed and considered for the routing process. Finally, other routing strategies and strategies for resource allocation can be developed, for example by improving the adaptive path search. It will be interesting to see whether these strategies can achieve better performance and avoid drawbacks of the strategies described in this work.

\section{Acknowledgements}

The author thanks Stefan Bodamer, Christoph Gauger, and Andreas Hess for valuable support to realise the simulation tool and all reviewers for their valuable comments.

\section{References}

[1] Optical Internetworking Forum, http://www.oiforum.com/.

[2] A. Adas, Traffic models in broadband networks, IEEE Commun. Magazine 35 (7) (1997) 82-89.

[3] G. Ash, Dynamic Routing in Telecommunications Networks, McGraw-Hill, New York, 1997.

[4] K. Bala, E. Bouillet, G. Ellinas, Benefits of 'minimal' wavelength interchange in WDM rings, in: Proceedings of the OFC' 97 , February 1997, pp. 120-121.

[5] S. Baroni, P. Bayvel, Key topological parameters for the wavelength-routed optical network design, in: Proceedings of the 22nd European Conference on Optical Communication (ECOC) '96, September 1996, pp. 2.277-2.280.

[6] R. Barry, P. Humblet, Models of blocking probability in all-optical networks with and without wavelength changers, IEEE J. Select. Areas Commun. 14 (5) (1996) 858-867.

[7] A. Birman, Computing approximate blocking probabilities for a class of all-optical networks, IEEE J. Select. Areas Commun. 14 (5) (1996) 852-857.

[8] C. Chen, S. Banerjee, A new model for optimal routing in all-optical networks with scalable number of wavelength converters, in: Proceedings of the IEEE Global Telecommunications Conference (Globecom '95), November 1995, pp. 993-997.

[9] R. Cruz, G. Hill, et al. (Eds.), Optical Networks (special issue), IEEE J. Select. Areas Commun. 14 (5) (1996).

[10] P. Demeester, M. Berger, M. Chbat, et al., Pan-European optical networking using wavelength division multiplexing, IEEE Commun. Magazine 35 (4) (1997) 82 88

[11] J. Färber, S. Bodamer, J. Charzinski, Statistical evaluation and modelling of Internet dial-up traffic, in: Proceedings of the SPIE Conference on 'Performance and Control of Network Systems III', September 1999, pp. $112-121$

[12] A. Feldmann, W. Whitt, Fitting mixtures of exponentials to long-tail distributions to analyze network performance models, Performance Evaluation 31 (1998) 245-279.

[13] M. Gagnaire, An overview of broad-band access technologies, Proc. IEEE 85 (12) (1997) 1958-1972.

[14] A. Girard, Routing and Dimensioning in Circuit-Switched Networks, Addison-Wesley, Reading, MA, 1990.

[15] H. Harai, M. Murata, H. Miyahara, Performance of alternate routing methods in all-optical switching networks, in: Proceedings of the IEEE Infocom '97, April 1997, pp. 517-525.

[16] H. Harai, M. Murata, H. Miyahara, Performance analysis of wavelength assignment policies in all-optical networks with limited-range wavelength conversion, IEEE J. Select. Areas Commun. 16 (7) (1998) 1051-1060.

[17] A. Hill, A. Saleh, K. Sato (Eds.), High-Capacity Optical Transport Networks (special issue), IEEE J. Select. Areas Commun. 16 (7) (1998).

[18] D. Hjelme, Importance of meshing degree on hardware requirements and capacity utilization in wavelength routed optical networks, in: Proceedings of the Third IFIP TC 6 Working-Conference on Optical Network Design and Modelling, February 1999, pp. 417-423.

[19] ITU-T Recommendation G.872, Architecture of optical transport networks, pre-published recommendation, February 1999.

[20] E. Karasan, E. Ayanoglu, Effects of wavelength routing and selection algorithms on wavelength conversion gain in WDM optical networks, IEEE/ACM Trans. Networking 6 (2) (1998) 186-196

[21] E. Karasan, E. Ayanoglu, Performance of WDM transport networks, IEEE J. Select. Areas Commun. 16 (7) (1998) 1081-1095.

[22] H. Kocher, M. Lang, An object-oriented library for simulation of complex hierarchical systems, in: Proceedings of the Object-Oriented Simulation Conference (OOS '94), January 1994, pp. 145-152.

[23] M. Koga, A. Watanabe, T. Kawai, K. Sato, Y. Ohmori, Large-capacity optical path cross-connect system for WDM photonic transport network, IEEE J. Select. Areas Commun. 16 (7) (1998) 1260-1269.

[24] M. Kovacevic, A. Acampora, Benefits of wavelength translation in all-optical clear-channel networks, IEEE J. Select. Areas Commun. 14 (5) (1996) 868-880. 
[25] P. Kühn, Approximate analysis of general queuing networks by decomposition, IEEE Trans. Commun. COM-27 (1) (1979) 113-126.

[26] K. Lee, V. Li, A wavelength-convertible optical network, J Lightwave Technol. 11 (5/6) (1993) 962-970.

[27] M. Listanti, M. Berdusco, R. Sabella, Optical path strategies in WDM all-optical networks: minimization of wavelength converters in optical cross connects, in: Proceedings of the IEEE Global Telecommunications Conference (Globecom '97), November 1997, pp. 583 587.

[28] M. Listanti, F. Middei, R. Sabella, S. Binetti, Routing strategies in WDM networks: the impact of transmission issues and network topology, in: Proceedings of the Third IFIP TC 6 Working-Conference on Optical Network Design and Modelling, February 1999, pp. 121-135.

[29] G. Maier, M. Martinelli, A. Pattavina, M. Scappini, Performance of WDM rings with wavelength conversion under non-Poisson traffic, in: Proceedings of the IEEE International Conference on Communications (ICC'99), paper S51-4, June 1999.

[30] A. Mokhtar, M. Azizoglu, Adaptive wavelength routing in all-optical networks, IEEE/ACM Trans. Networking 6 (2) (1998) 197-206.

[31] V. Paxson, S. Floyd, Wide area traffic: the failure of Poisson modelling, IEEE/ACM Trans. Networking 3 (3) (1995) 226--244.

[32] C. Qiao, M. Yoo, Optical burst switching (OBS) -- a new paradigm for an Optical Internet, J. High Speed Networks 8 (1) (1999) 69-84

[33] B. Ramamurthy, B. Mukherjee, Wavelength conversion in WDM networking. IEEE J. Select. Areas Commun. 16 (7) (1998) 1061-1073.

[34] R. Ramaswami, K. Sivarajan, Routing and wavelength assignment in all-optical networks, IEEE/ACM Trans. Networking 3 (5) (1995) 489 500.

[35] R. Ramaswami, K. Sivarajan, Optical Networks - A Practical Perspective, Morgan Kaufmann, Los Altos, CA, 1998.

[36] K. Sato, S. Okamoto, H. Hadama, Network performance and integrity enhancement with optical path layer technologies, IEEE J. Select. Areas Commun. 12 (1) (1994) 159-170.

[37] K. Sato, Advances in Transport Network Technologies: Photonic Networks, ATM, and SDH, Artech House Publishers, 1996

[38] J. Späth, S. Bodamer, Routing strategies for photonic networks under dynamic traffic conditions, in: Proceedings of the NOC' 98 (Network \& Optical Communications), vol. 3 (Technology and Infrastructure), June 1998, pp. 174 177.

[39] J. Späth, S. Bodamer, Routing of dynamic Poisson and non-Poisson traffic in WDM networks with limited wavelength conversion, in: Proceedings of the 24th European Conference on Optical Communication (ECOC) '98, September 1998 , pp. $359-360$.

[40] J. Späth, Design parameters for dynamic routing in WDM cross-connect networks, in: Proceedings of the Third IFIP TC 6 Working-Conference on Optical Network Design and Modelling, February 1999, pp. 107-120.
[41] J. Späth, Resource allocation for dynamic routing in WDM networks, in: Proceedings of SPIE Conference on All-Optical Networking 1999: Architecture, Control, and Management Issues, September 1999, pp. 235-246.

[42] K. Struyve, P. Demeester, Dynamic routing of protected optical paths in wavelength routed and wavelength translated networks, in: Proceedings of the 23rd European Conference on Optical Communication (ECOC) '97, paper TU 3, September 1997.

[43] S. Subramaniam, M. Azizoglu, A. Somani, All-optical networks with sparse wavelength conversion, IEEE/ACM Trans. Networking 4 (4) (1996) 544-557.

[44] S. Subramaniam, M. Azizoglu, A. Somani, On the optimal placement of wavelength converters in wavelength-routed networks, in: Proceedings of the IEEE Infocom '98, March 1998, pp. 902-909.

[45] S. Subramaniam, A. Somani, M. Azizoglu, R. Barry, A performance model for wavelength conversion with nonPoisson traffic, in: Proceedings of the IEEE Infocom '97, vol. 1, April 1997, pp. 500 507.

[46] S. Thiagarajan, A. Somani, An efficient algorithm for optimal wavelength converter placement on wavelengthrouted networks with arbitrary topologies, in: Proceedings of IEEE Infocom '99, vol. 2, March 1999, pp. 916-923

[47] T. Tripathi, K. Sivarajan, Computing approximate blocking probabilities in wavelength routed all-optical networks with limited-range wavelength conversion, in: Proceedings of the IEEE Infocom '99, vol. 1, March 1999, pp. 329-336.

[48] W. Van Parys, B. Van Caenegem, P. Demeester, Reduction of blocking in WDM networks through a biased routing approach, in: Proceedings of the OFC '98, February 1998, p. 94.

[49] K. Venugopal, E. Rajan, P. Kumar, Impact of wavelength converters in wavelength routed all-optical networks, Comput. Commun. 22 (3) (1999) 244-257.

[50] K. Venugopal, M. Shivakumar, P. Kumar, A heuristic for placement of limited range wavelength converters in alloptical networks, in: Proceedings of the IEEE Infocom ' 99 , vol. 2, March 1999, pp. 908-915.

[51] A. Watanabe, S. Okamoto, K. Sato, WDM optical pathbased robust IP backbone network, in: Proceedings of the OFC 99, February 1999, pp. Tu56-Tu58.

[52] M. Yamaguchi, K. Arai, I. Nakamura, Outline of NEC's transmission systems - photonic IP networking, NEC Res. Dev. 40 (1) (1999) 1-7.

[53] J. Yates, J. Lacey, D. Everitt, M. Summerfield, Limitedrange wavelength translation in all-optical networks, in: Proceedings of the IEEE Infocom '96, vol. 3, March 1996. pp. 954961

[54] J. Yates, M. Rumsewicz, J. Lacey, Wavelength converters in dynamically-reconfigurable WDM networks, IEEE Communications Survey, http://www.comsoc.org/pubs/ surveys, Second Quarter 1999.

[55] Y. Zhu, G. Rouskas, H. Perros, Blocking in wavelength routing networks, Part II: Mesh topologies, in: Proceedings of the 16th International Teletraffic Congress (ITC-16), June 1999, pp. 1321-1330. 


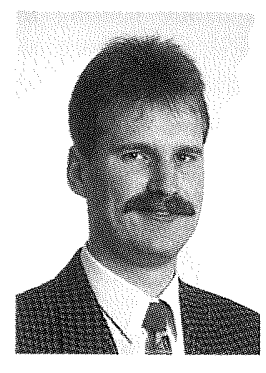

Jan Späth was born in 1970 and received the Dipl.-Ing. degree in Electrical Engineering with Distinctions from the University of Stuttgart, Germany, in 1994. Since 1994, he has been a member of scientific staff at the Institute of Communication Networks and Computer Engineering at the University of Stuttgart. His research interests include network planning and performance evaluation, especially for photonic networks. He has been participating in several national and European projects in this field. In ad-

dition, he has been a lecturer on computer communication networks at the Berufsakademie Stuttgart since 1995. 